\title{
Detecting Vacancy-Induced Strain Field Oscillations via Deep Learning
}

Chia-Hao Lee, Abid Khan, Di Luo, Tatiane Santos, Chuqiao Shi, Blanka Janicek, Sangmin Kang, Wenjuan Zhu, Nahil Sobh, Andre Schleife, Bryan Clark and Pinshane Huang

University of Illinois at Urbana-Champaign, Urbana, Illinois, United States

As atomic-resolution datasets are now being routinely acquired by advanced instruments, new approaches for data extraction are necessary to fully exploit large datasets and utilize information that might be normally overlooked and discarded. Because two-dimensional (2D) materials are only a single unit cell thick, they provide opportunities to characterize almost every atom and their responses to atomic defects. Yet, high-precision characterization of single-atom defects remains challenging because 2D materials are beam-sensitive and are weak electron scatterers. Existing imaging techniques struggle to provide sufficiently high signal-to-noise ratio (SNR) and spatial resolution to measure the structure of single-atom defects without inducing electron beam damage. In our work, we apply deep learning techniques based on fully convolutional networks (FCN) to achieve sub-pm precision measurements of the positions of single atom in aberration-corrected scanning transmission electron microscopy (STEM) images [1]. We demonstrate our approach using an alloyed 2D transition metal dichalcogenide (TMDC), monolayer WSe2-2xTe2x. We show that deep learning enables the first direct measurement of strain field oscillations around vacancies, a phenomenon which has been predicted since the mid 1950s [2,3] but never experimentally imaged.

First, we construct FCN models to identify different types of chalcogen defects, including one or two Te substitutions, and single or double Se vacancies in monolayer WSe2-2x $\mathrm{Te}_{2 \mathrm{x}}$ as shown in Figure 1a. By combining hundreds of single images of nominally identical atomic defects (Figure 1b-e), we generate class-averaged images with high SNR using rigid registration. These high SNR images allow us to measure 2D atomic coordinates of single atoms with up to $0.3 \mathrm{pm}$ precision, a 20 -fold improvement over the original images. Next, we measure the displacement and strain fields for each defect type. Figure 2a shows magnified 2D displacement vectors overlaid on class-averaged image of single Se vacancy. We used the displacement field to calculate the $2 \mathrm{D}$ strain tensor components $\varepsilon_{\mathrm{xx}}, \varepsilon_{\mathrm{yy}}$, and the dilation $\left(\varepsilon_{\mathrm{xx}}+\right.$ $\left.\varepsilon_{y y}\right)$ as shown in Figure $2 b-d$. We next compare these experimental strain fields to those calculated by elastic continuum theory using the 2D version of Eshelby's inclusion model [4] (Figure 2e-g). While the elastic model captures most of the behavior near the defect core, the experimental strain fields (Figure 2bd) reveal fine features that cannot be explained by elastic theory. To investigate these differences in more detail, line profiles of Eyy derived from experiment, elastic theory, and density functional theory (DFT) are shown in Figure $2 \mathrm{~h}$ respectively. We observed long-range oscillations in both experiment and DFTderived strain fields which are not present in the elastic model. Moreover, we obtained excellent agreement between experiment and DFT, particularly for the locations of strain field minima and maxima. Direct observation of these oscillating strain fields in 2D materials paves the way to a better understanding of the complex response of each atom to local perturbations. More broadly, our methods should be particularly useful for studying 2D materials and other radiation-sensitive crystals, and in principle can be applied to any atomic resolution electron microscopy datasets including spectral imaging and 4D STEM [5]. 

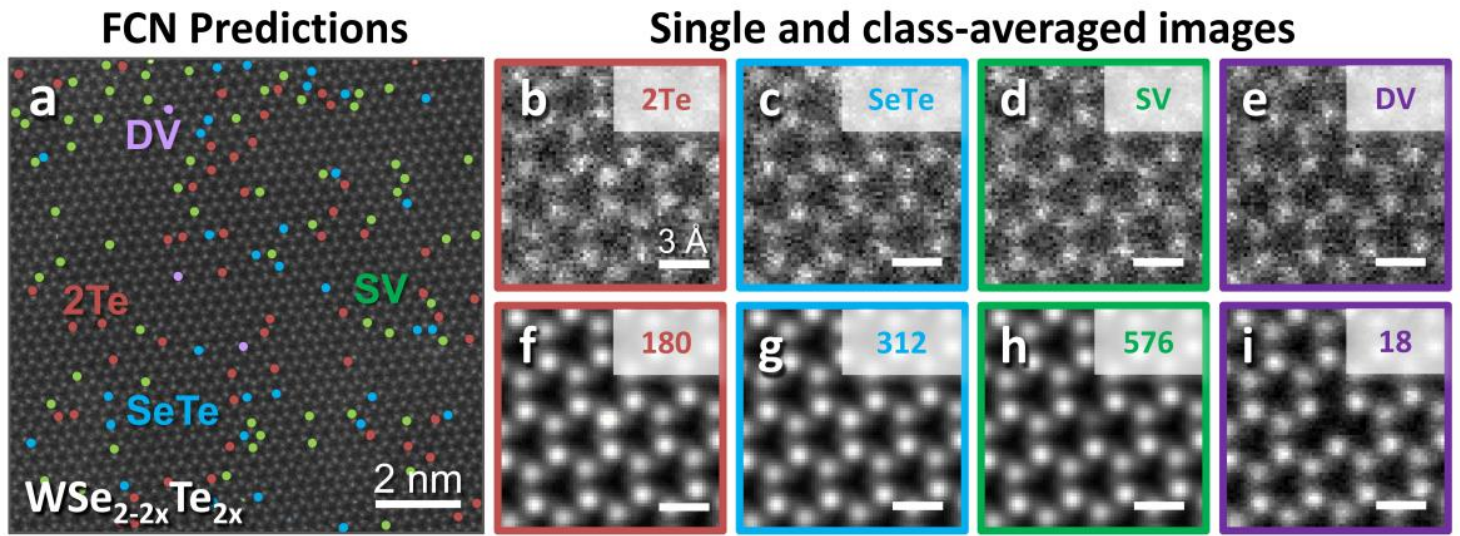

Figure 1. Figure 1: Deep learning-enabled defect identification and high signal-to-noise class averaged images. (a) Chalcogen site defects identified by fully convolutional networks (FCN) overlaid on annular dark-field STEM image of WSe2-2xTe2x . Labels indicate one or two Te substitutions (SeTe and 2Te, respectively) and single or double Se vacancies (SV and DV). (b-e) Representative single images of FCNidentified defects sectioned from (a). By aligning and summing many equivalent lattice sites using rigid registration, we produce high SNR, class-averaged images (f-i) from nominally identical point defects. The number of images summed is labeled at the top right corner of each image. Adapted from reference [1].

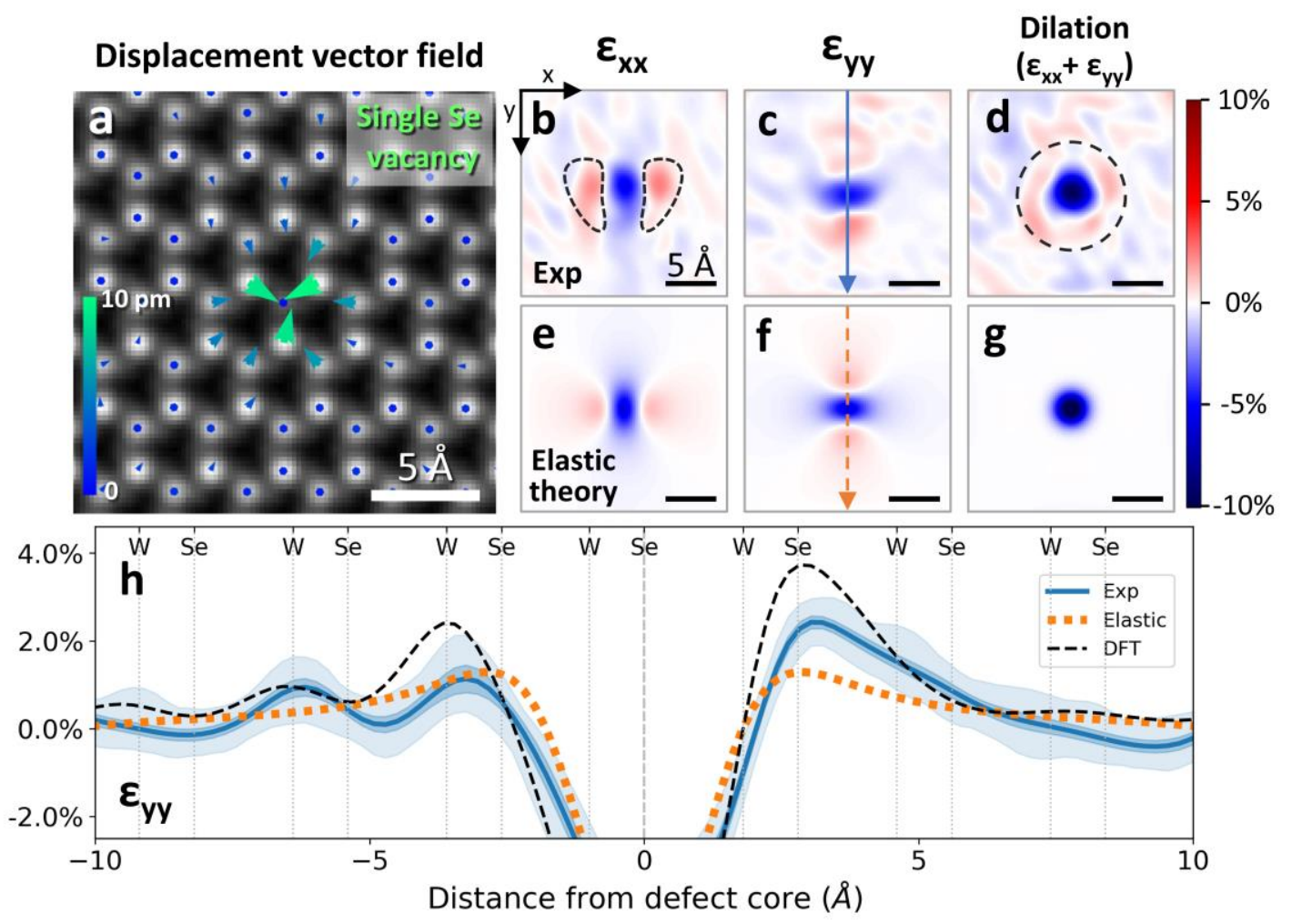

Figure 2. Figure 2: Displacement and strain fields of a single Se vacancy. (a) Two-dimensional displacement vectors overlaid on class-averaged image of single Se vacancy. The vectors are enlarged 18 times for visibility. (b-d) Experimental strain fields calculated from the derivative of displacement field in (a). (e-g) Best-fit strain fields calculated by elastic theory using Eshelby's inclusion model. (h) Line 
profiles of experimental, elastic theory, and DFT-derived eyy across the vacancy, as marked by the solid (blue) and dashed (orange) arrows on (c and f). The shaded regions of the experimental line profile correspond respectively to \pm 1 standard deviation $( \pm 0.2 \%)$ and the full-range of the experimental distribution of strain values. In contrast to the monotonically decaying strain field predicted by continuum elastic theory, both experimental and DFT profiles show oscillations in the strain field. Adapted from reference [1].

\section{References}

1. C.-H. Lee, et al., arXiv:2001.08233 (2020)

2. H. Kanzaki, Journal of Physics and Chemistry of Solids 2 (1957), p. 24-36

3. G. L. Hall, Journal of Physics and Chemistry of Solids 3 (1957), p. 210-222

4. A. L. Kolesnikova, et al., Physics of the solid state 56 (2014), p. 2573-2579

5. This work was supported by the AFOSR under award number FA9550-7-1-0213, DOE award number DE-SC0020190, ONR grant numbers NAVY N00014-17-1-2973 and N00014-18-1-2605. This research is part of the Blue Waters sustained-petascale computing project, which is supported by NSF awards OCI0725070 and ACI-1238993 and the state of Illinois. This work was carried out in part in the Materials Research Laboratory at UIUC. 